\title{
A New Approach to Automatic Radar Tracking with and without Supplementing AIS Data in Tokyo Bay
}

\author{
NGUYEN Minh Duc* and TAMARU Hitoi ${ }^{* *}$
}

\begin{abstract}
Marine traffic observation is vitally important for congested water with special geographical characteristics like Tokyo bay. Therefore, the study aims at introducing an approach of automatic radar tracking with better accuracy and efficiency, and a method of using AIS data as supplement to improve the stability, quality of auto-tracking. To reach the targets, a Relating-Function is suggested. The tracking results are later compared with manual tracking results to ensure the accuracy and prove to be promising. Approach with supplementing AIS data would be very useful as requirements for carrying class B AIS are coming into effects.
\end{abstract}

Keywords: Radar System, Tokyo Bay, Marine Traffic, AIS, Automatic tracking, Relating-Function.

\section{Introduction}

Tokyo Bay has been well known to be one of the most congested marine traffic areas around the world, with the number of ships navigating in/out or inside the bay to be well over 1000 ships per day. In "Rush Hour", there are as many as over 100 ships navigating inside the bay simultaneously. Thus, the close and efficient observation of the traffic is of great importance as far as safety of navigation, environment protection and more efficient traffic service are concerned.

A Radar/AIS network system for observation of marine traffic in the bay has been constructed, including 2 radar stations namely Kawasaki station and Yokosuka station. The AIS transponder is also installed at Kawasaki station, giving the composite picture of traffic inside the bay in real time. The data observed from these stations are transferred and saved at the main server in TUMSAT to be analyzed.

Basing on the structures, some different researches have been conducted, including but not to be limited to:

Liu et al 2004, 2005, 2006 with their research on AIS Data of Tokyo Bay, has made an effort in using AIS data for traffic observation ${ }^{(1)}$. However, there was just limited number of ships with AIS observed, and the reliability and accuracy of AIS data is sometimes in doubt and difficult to be recognized.

Okano et al 2004, 2005, 2006, in their researches on Radar Network System for observing and analyzing marine traffic in Tokyo bay, used Radar images for automatic tracking and estimating the target length ${ }^{(2)}$. The limitation of this approach is mainly the low probability of auto-tracking.

Therefore, to find the accurate information of vessels moving inside the bay for traffic analyzing purpose, in this paper, we aimed at:
- Firstly, implementing a method of automatic radar tracking that give better tracking probability (percentage of targets tracked), especially in noisy condition.

- Secondly, as AIS data are available for some ships, we would like to introduce a method of using these AIS data together with Radar Image to produce more accurate and stable tracking results. That means to avoid the possibility of "shift target" or "lost target" while tracking the ship on Radar images.

\section{A new approach to automatic Radar tracking without AIS Data}

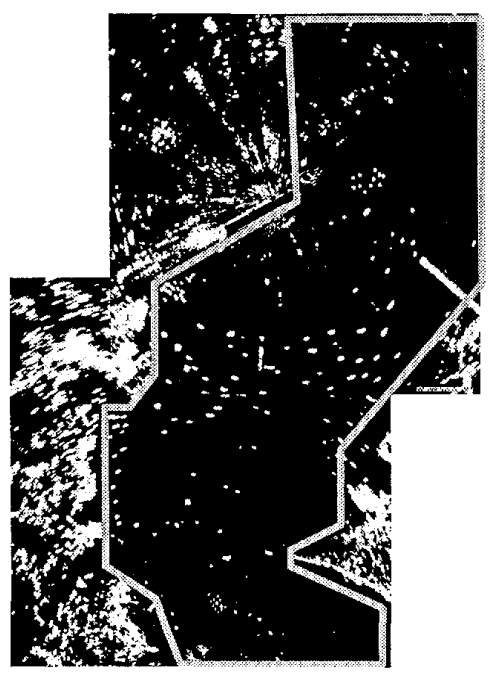

Fig.1 Radar pictures and observing area

\subsection{General principle}

Radar screen of each station is saved in the form of a $1024 \times 1024$ bitmap and referred to as radar picture.

The Radar pictures of two Radar stations, after

\footnotetext{
* Student member: Tokyo University of Marine Science and Technology (2-1-6, Etchujima, Koto ku, Tokyo 135-8533).

** Member: Tokyo University of Marine Science and Technology (2-1-6, Etchujima, Koto ku, Tokyo 135-8533).
} 
receiving, are imposed on each other according to a time based fundamental to produce the composite radar picture of Tokyo Bay. Removing coastline and on shore targets, the area of observation is that marked on Fig. 1 .

From the picture, a set of "Image Positions" can be extracted. In this paper, a Position is defined to include its coordination $(x, y)$, number of brink pixels and a matrix containing luminance value (from 0 to 255) of each pixel in that Position ${ }^{(2)}$.

From 3 consecutive Image Positions Sets, moving targets and non-moving targets can be selected.

\subsection{Relating-Function}

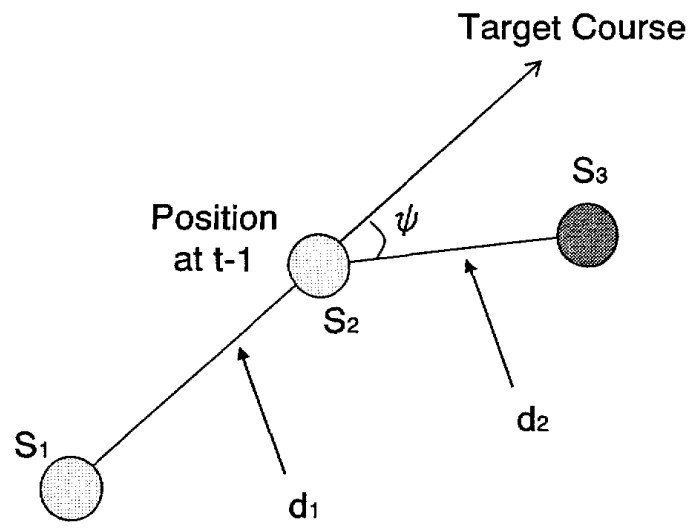

Position

at $\mathrm{t}-2$

Fig.2 Three positions Relationship

To evaluate moving target from 3 consecutive image positions, a "Relating-Value" is defined. Formula for calculating 3 positions Relating-Value is

$$
\begin{aligned}
& R= d_{m d f}+f \times S_{m d f}-\cos (\psi) \\
& d_{2} \leq d_{1} \Rightarrow d_{m d f}=\frac{d_{1}}{d_{2}}-1 \\
& d_{2}>d_{1} \Rightarrow d_{m d f}=\frac{d_{2}}{d_{1}}-1 \\
& 2 \times S_{3}>S_{1}+S_{2} \Rightarrow S_{m d f}=\frac{2 \times S_{3}}{S_{1}+S_{2}}-1 \\
& 2 \times S_{3} \leq S_{1}+S_{2} \Rightarrow S_{m d f}=\frac{S_{1}+S_{2}}{2 \times S_{3}}-1
\end{aligned}
$$

Where:

- R: the Relating-Value to be calculated.

$-d_{\text {mdf }}$ : modified distance ratio, representing the relationship in distance of the 3 positions.

$-S_{\text {mdf }}$ : modified pixel ratio, representing the size relationship of these 3 positions. $S_{i}$ is the pixel number of position $i$.

$-\psi$ is the difference in relative bearings between position at $(\mathrm{t}-2)$ to position at $(\mathrm{t}-1)$, and position at $(\mathrm{t}-1)$ to position at $\mathrm{t}$ respectively.

In formula (1), the ratios have been modified to keep them non-negative in all cases. Because reliability of distance and pixel ratio are different, depending on the existence of noise on the image, a factor $\mathbf{f}$ is included in the Relating-Function to weight these differences. Value of $f$ will then be chosen basing on observation results.

As a common knowledge of navigators, the most reliable case of confirming the existence of a moving target is when the 3 image positions are distributed equally, on a straight line, and of the same size. In this case, each values of equation (1) are

$$
\mathrm{d}_{1}=\mathrm{d}_{2}, \mathrm{~S}_{1}=\mathrm{S}_{2}, \psi=0 \Rightarrow \mathrm{R}=-1
$$

The larger $\mathrm{R}$ becomes, the less possible 3 image positions are those of a moving target.

For checking the validity of the suggested function, Density function of pixel and distance ratios that were calculated from actual observation moving targets were taken as shown in Fig.3.

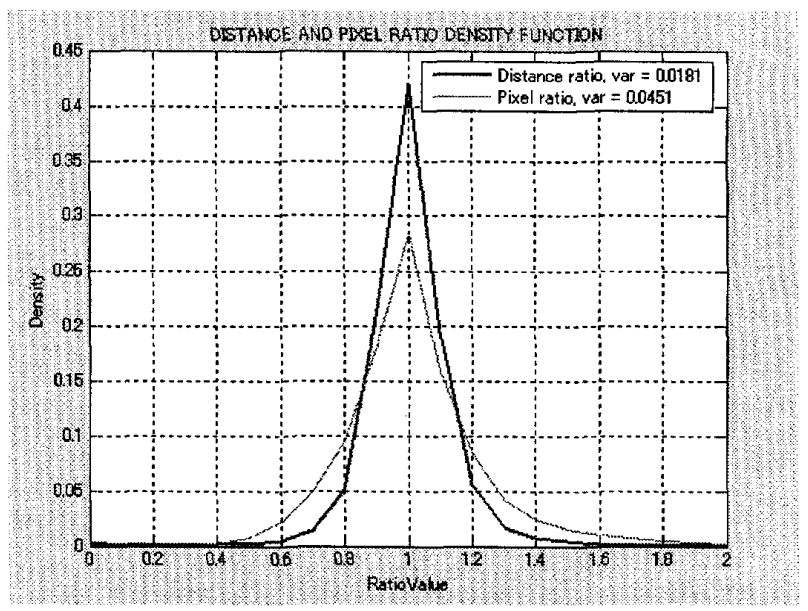

Fig.3 Distance and Pixel Ratio density function

Distance Ratio $=d_{1} / d_{2}$ (see Fig. 2)

Pixel-Ratio $=2 \mathrm{xS}_{3} /\left(\mathrm{S}_{1}+\mathrm{S}_{2}\right)$

As seen clearly from Fig. 3, variance of the distance ratio is much less than that of pixel ratio, giving a base for choosing $f$ to be less than 1 . Experiments have shown that choice of this factor to be from 0.2 to 0.5 is acceptable.

In Fig.4, distribution functions of the Relating-Functions have been drawn as a reference for choices of threshold values. In this figure, value of factor $f$ in formula (1) has been chosen to be 0.1 to 0.6 , giving different values for relating function.

For larger value of $f$, Relating-Value becomes smaller and it is difficult to determine if the 3 
points are 3 consecutive images of a ship, because this value is nearer to that of random noise.

However, if $\mathbf{f}$ is too small, there is risk of wrong determination the target, because the size of image position is not sufficiently taken into account.

In this study, $\mathrm{f}$ is suggested as 0.3 . For this value, relating-value of more than $90 \%$ of observations is less than -0.60 . Therefore, when the moving target has been found, value for Relating-Function is chosen to be -0.6 for continuous tracking. It means, if the relating-value of a new position, compared with 2 previous positions of the ship is less than -0.6 , this position can be taken as ship's new position. For deciding the existence of a moving target from 3 image positions, the Threshold value is set to be -0.85 . The small value is chosen to avoid taking random noise as target.

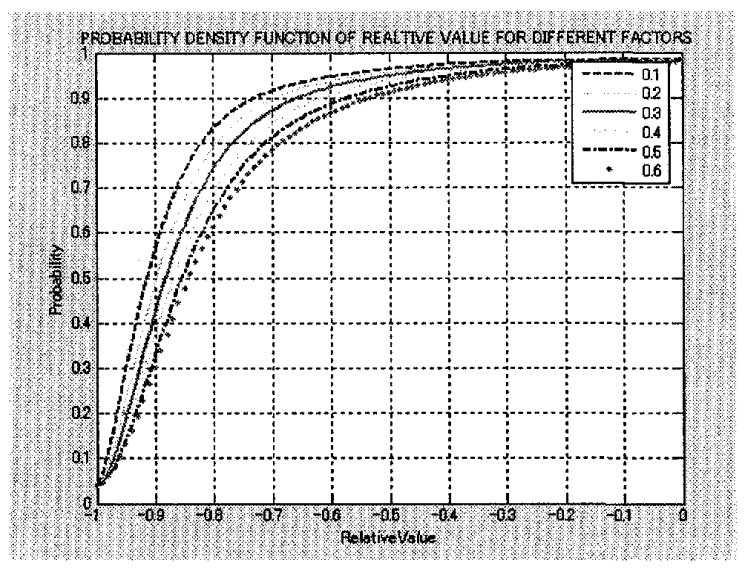

Fig.4 Relating-Value distribution

Similarly, Relating-Function can be applied also to targets in overlapping. In this case, pixel ratio should be removed, i.e. $\mathrm{f}$ is set to 0 , because the pixel number of position after dividing is not reliable.

\subsection{Tracking moving target}

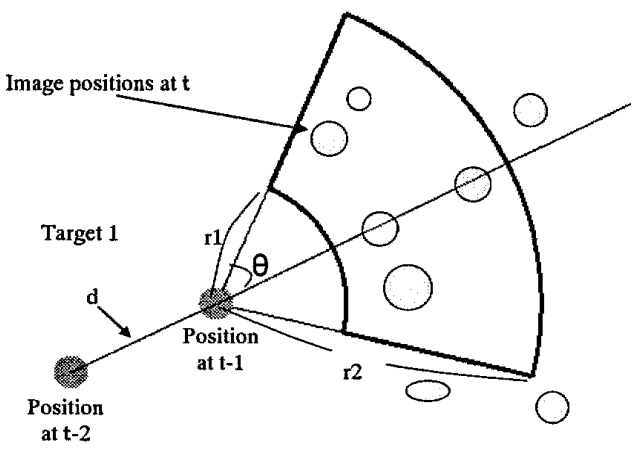

Fig.5 Tracking moving target principle

In Fig. 5, positions of a moving target (target 1) are superposed on radar picture at $t$. Then, its position at $t$ can be determined from its positions at $\mathrm{t}-2, \mathrm{t}-1$ and image positions at $\mathrm{t}$, using the following tracking procedure:
- Step 1: Define the possible position set for that target. The possible positions are those lying in the searched area as shown in Fig.2. Considering the normal speed of ships and possible speed change (as target speed is not constant) and course change in one time interval (here 1 minute), radiuses $\left(r_{1}, r_{2}\right)$ and limiting angle were decided to be:

$$
\begin{aligned}
& \mathrm{r} 1=0.5 \times \mathrm{d} ; \mathrm{r} 2=2.0 \times \mathrm{d} \\
& \theta=60^{\circ}
\end{aligned}
$$

In equation (2), $\mathrm{d}$ is the distance between positions at $\mathrm{t}-2$ and $\mathrm{t}-1$ of the target, $\theta$ is the limiting angle.

- Step 2: Use formula (1) for target's positions at $t-2, t-1$ and every one position in the set found in step 1 to determine the most possible position and decide if it can be taken as position of the target.

Similarly, these 2 steps can be used to find a new target if we consider an image position at $\mathrm{t}-2$, an image position at $\mathrm{t}-1$ and the positions set at $\mathrm{t}$.

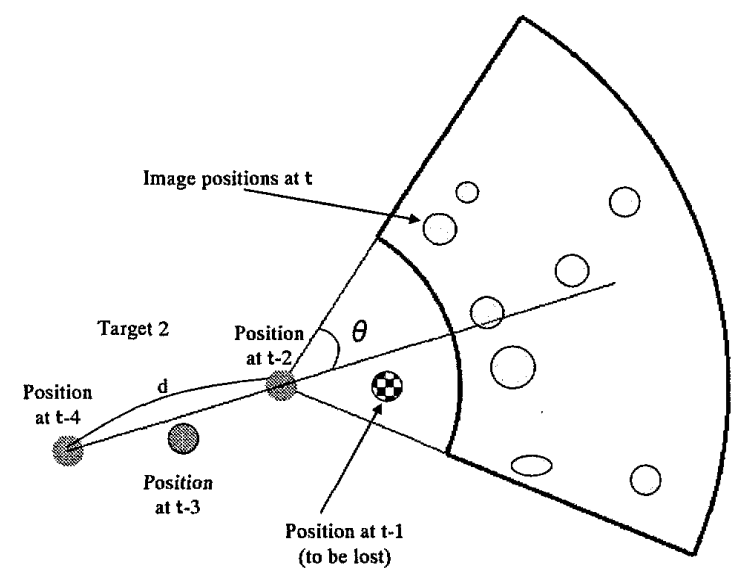

Fig.6 Tracking lost target

If a target lost its position at $\mathrm{t}-1$ (lost target), its current position can be determined in a similar manner by using its positions 2 and 4 steps ahead instead, together with current image positions as shown in Fig.6, where, due to different reason, target position at $\mathrm{t}-1$ can not be found.

\subsection{Tracking Possible Overlapping Couples}

Because of limitation of picture resolution, there are cases where images of two or more targets overlap or may overlap each others. In this study, just two targets overlapping case was taken into consideration and these two targets are hereafter referred to as possible overlapping couple. The problem of 3 or more targets overlapping is pretty rare and very complicated to solve, therefore not to be mentioned.

Procedure for solving this case is as followings:

- Step1: Define the possible positions set for each target of the couple. The possible positions sets are defined the same way as descried in 2.3 .

-Step 2: Consider the combined set to confirm the overlapping case. 
-Step 3: If the overlapping case is confirmed, the overlapping position will be divided into 2 positions by a line perpendicular to the relative bearing to two dead reckoning positions of the two targets (Fig.7, lower part).
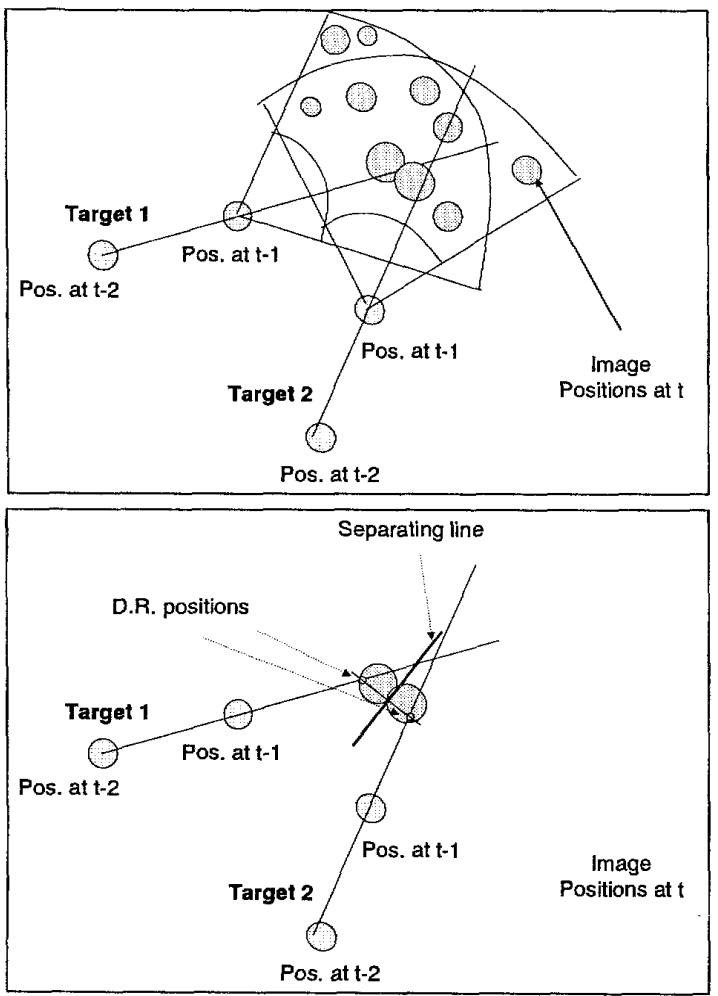

Fig. 7 Overlapping Targets

It is considered the case when there are no suitable positions for each target and/or two targets share the shame most suitable position, which is larger than both the positions of targets.

-Step 4: Apply the Relating-Function as expressed in 2.2 to each position in the combined set, determine the most possible position for each target and decide if this position can be taken as position of the target.

\subsection{Solving non-moving target}

Non-moving target is determined by checking the overlapping case of 3 image positions, together with considering the Relating-Value of 3 mutual overlapping positions.

\subsection{Overall tracking procedures}

To achieve the best possible results, tracking program should follow a certain procedure as listed below:

\subsubsection{Start Tracking}

-Step1: Read 3 first pictures into 3 image positions set respectively.

-Step 2: Define the non-moving targets from image positions sets.

-Step 3: Define moving targets.

-Step 4: Classify moving targets into normal moving targets set and possible overlapping couples set.

-Step 5: Shift the positions set backward.

\subsubsection{Continue Tracking}

-Step 1: Read a new radar picture into the newest position set.

-Step 2: Determine image positions of existing non-moving targets.

-Step 3: Define newly appeared non-moving targets.

-Step 4: Determine positions of existing moving targets not in possible overlapping couples.

-Step 5: Solve the possible overlapping couples.

-Step 6: Define newly appeared moving targets.

-Step 7: Classify moving targets into normal moving targets set and possible overlapping couples set.

-Step 8: Shift the positions set backward.

\subsection{Tracking results}
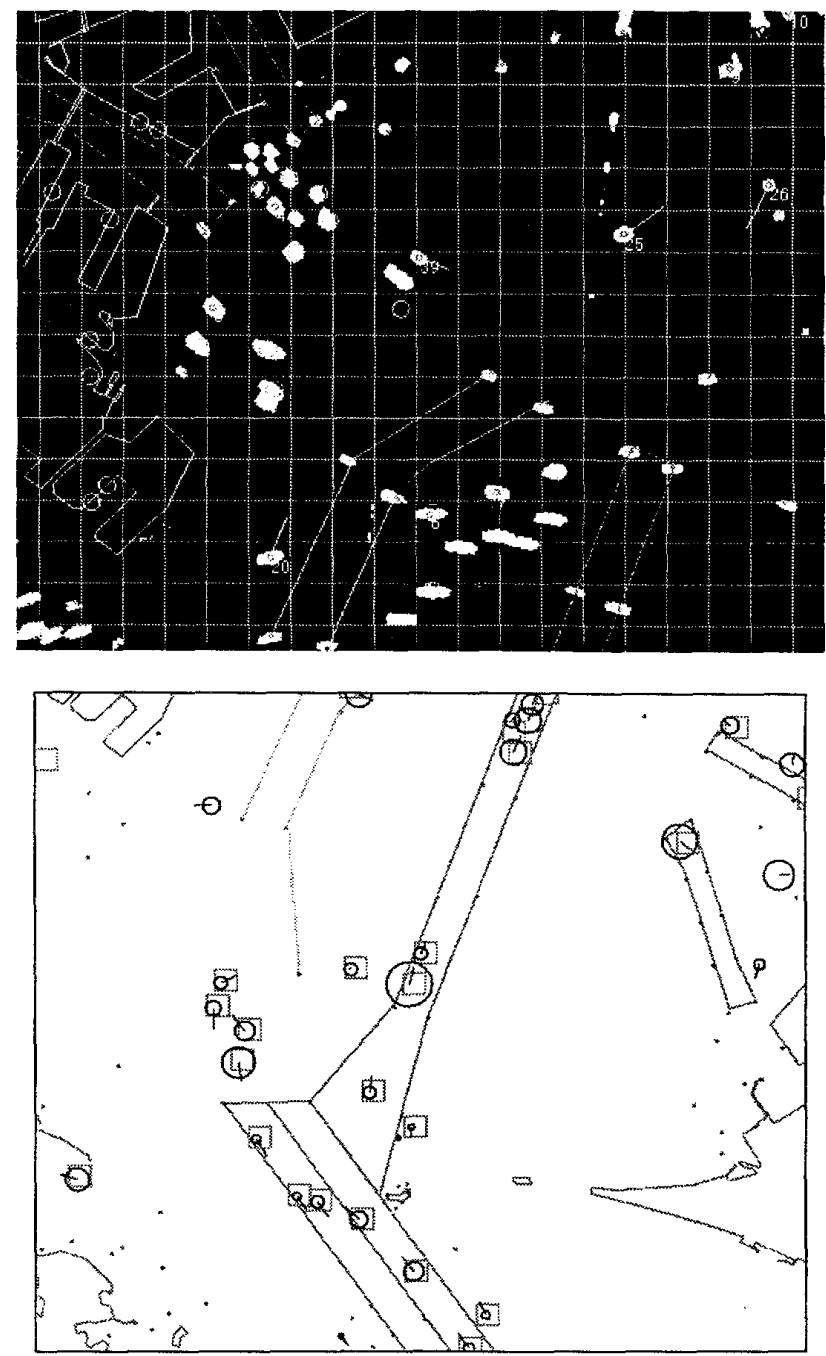

Fig.8 Screens for Auto-tracking and Results checking

- $\square$ : manually tracked targets

- $\bigcirc$ with velocity vector: targets automatically tracked by this approach. 
The approach has been applied for automatic radar tracking with several days of radar pictures of 2006. The 2006 observing data has been intentionally chosen on purpose of comparing with results of previous approach and as well as with available manual tracking results.

To ensure the accuracy, the results are first visually compared with those of manual tracking. Comparing by user graphics interface is shown in lower screen of Fig. 8.

Auto-tracked targets with no equivalent manually tracked one need further checking, using auto-tracking interface (upper screen of Fig.8). Targets are indicated with their ID-number and trajectory lines.

Once accuracy has been ensured, tracking results are compared to results of previous methods and manual tracking results to prove the efficiency.

Data of 2 randomly chosen days of Apr. 2006 have been used, giving the comparison graphs (see Fig.9).
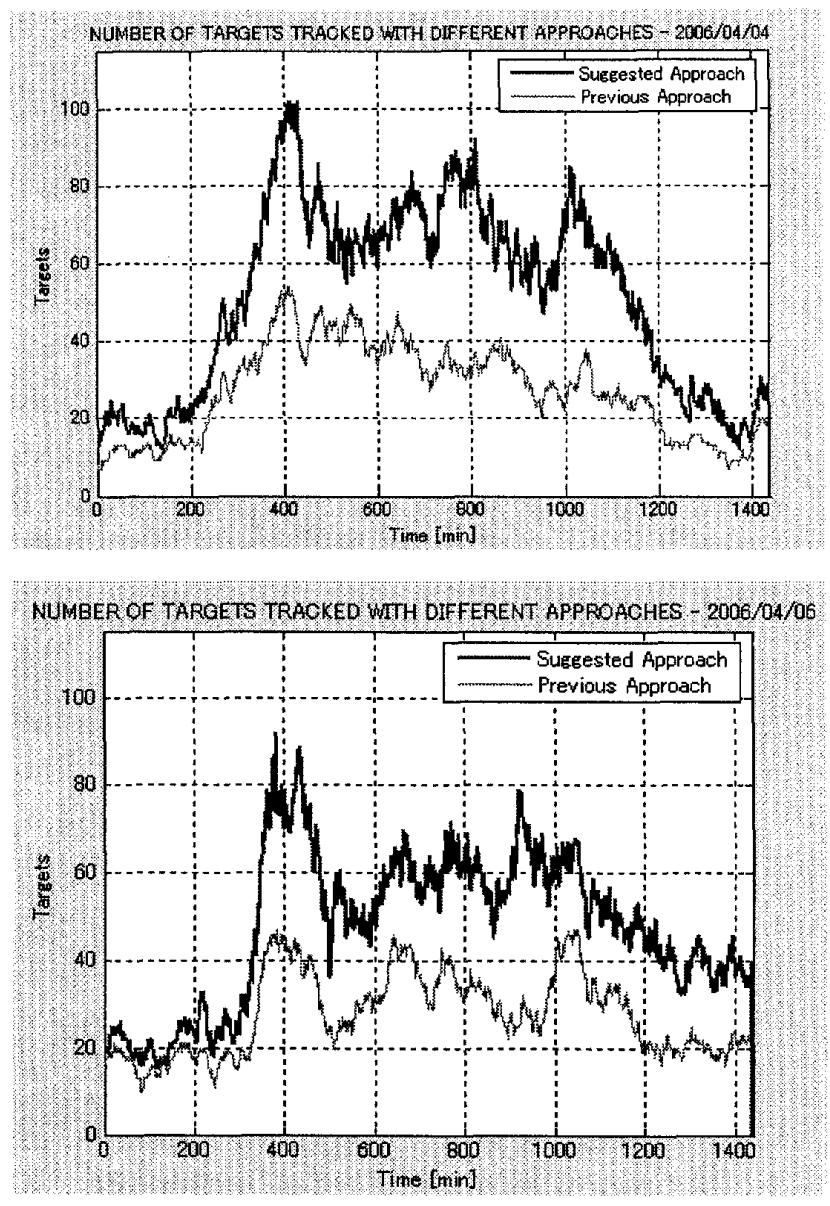

Fig. 9 Tracking results, in comparison with previous approach

In comparison with previous approach, the number of targets tracked automatically has been increased drastically, and, as shown in Fig.9, many targets have been tracked automatically before they are recognized by manual observation.
Apart from that, there are cases where it is difficult to affirm the existence of a moving target, especially for those with small and weak radar reflection. The auto-tracking program can detect these at the price of possibility of taking noise as targets.

\section{An approach of automatic Radar tracking with supplementing AIS Data \\ 3.1 AIS-Radar Target}

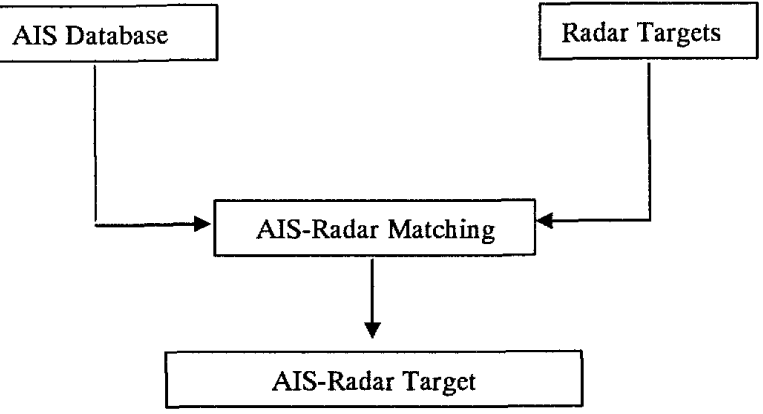

Fig.10 AIS-Radar match

Data provided by AIS, if properly used, would be an immense source of information for more efficient tracking and marine traffic observing.

Once available, AIS targets from AIS database are matched with targets automatically tracked from radar pictures to define AIS-Radar targets. In this paper, AIS-Radar targets are the radar targets which have been confirmed to be the image of ship equipped with AIS (see Fig.10). Then, AIS data can be used to facilitate tracking.

\subsection{Modified Relating-Function}

For tracking AIS-Radar targets, Relating-Function as defined in 2.2 is slightly modified to take advantage of the data provided by AIS.

$$
\begin{aligned}
& R=d_{m d f}+f \times S_{m d f}-\cos (\psi) \\
& d_{2} \leq d \Rightarrow d_{m d f}=\frac{d}{d_{2}}-1 \\
& d_{2}>d \Rightarrow d_{m d f}=\frac{d_{2}}{d}-1
\end{aligned}
$$

Where:

$d=A I S_{-} S o g[p x l / \mathrm{min}] \times 1[\mathrm{~min}]:$ the distance covered in 1 time unit.

$\Psi$ : the difference between AIS_COG and direction to the position that needs checking. 


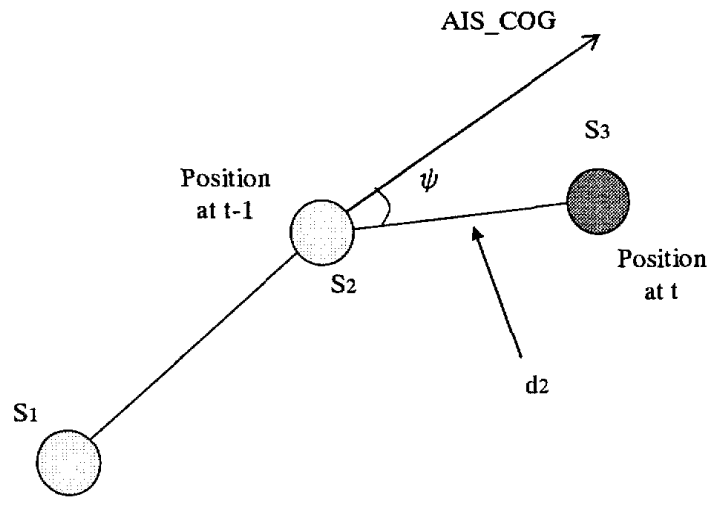

Position at

$\mathrm{t}-2$

Fig.11 3 positions Relationship with AIS data

Distribution function for Relating-Value of AIS-Radar Target is shown in Fig.12.

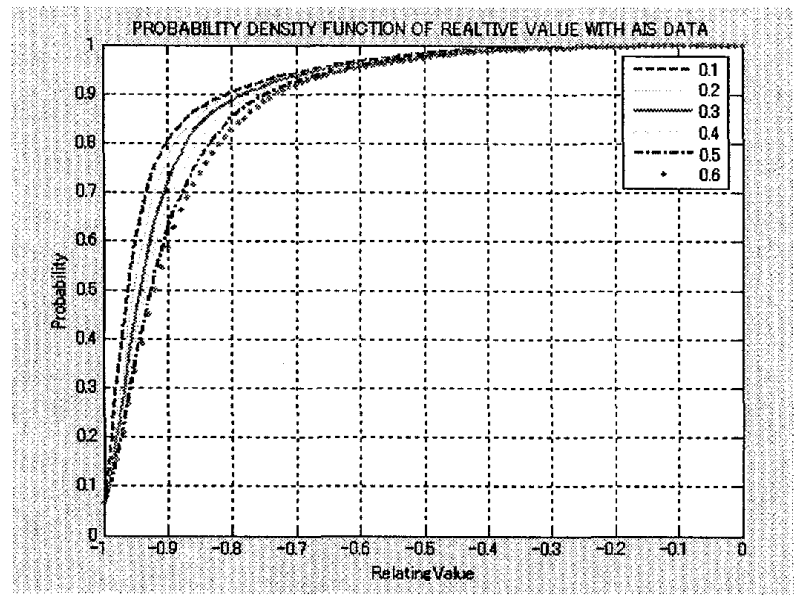

Fig.12 Relating-Value distribution function of AIS-Radar Targets

In comparison with Relating-Value distribution in Fig.4, the convergence appears to be much better, enabling the elimination of the possibility of taking noise as positions for targets.

For $\mathrm{f}=0.3$ in formula (3), relating-value of 3 positions of an AIS-Radar target in $90 \%$ is less than -0.8 , thus the risk of wrong tracking becomes less.

\subsection{Tracking AIS-Radar targets}

Procedure of tracking AIS-Radar target is almost similar to that of normal moving target as mentioned in 2.3. However, the search area is now chosen to be a circle with center defined from AIS-Radar targets previous position, together with their AIS_COG and AIS_SOG, as in Fig.13.

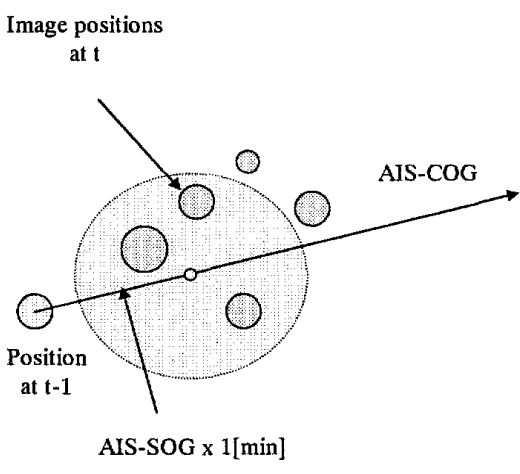

Fig13 Tracking AIS-Radar Targets

Radius of the circle can be chosen rather randomly to be about 0.7 times of distance run in 1 time unit (here 1 min.).

Then, formula (3) is used to find the most possible one and decide if this position can be taken as position for the target. In this study, a threshold value of -0.80 is used.

If no suitable position is available, then, overlapping case should be born in mind.

For this case, current position of the target (Position at $\mathrm{t}-1$ ) can be shifted to center of the circle to find the overlapping position and consider this position for position of the target (see Fig.14)

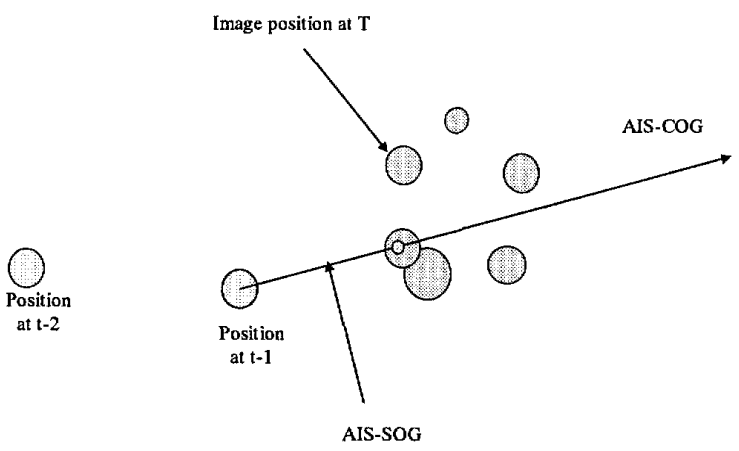

Fig.14 Overlapping Case of AIS-Radar targets

\subsection{Tracking results}

The approach with supplementing AIS data has also been applied for several days of AIS and Radar data. Tracking results were firstly visually check to ensure the accuracy (see Fig.15) and then compared with AIS data to show the efficiency.

Compared with AIS data, probability of AIS carrying ships matched and tracked automatically is more than $80 \%$ for all days of experiment $(82 \%$ and $84 \%$ respectively in Fig.16). Using approach with AIS data, these targets are tracked continuously and stably, without risk of target swapping (switching) phenomenon. 


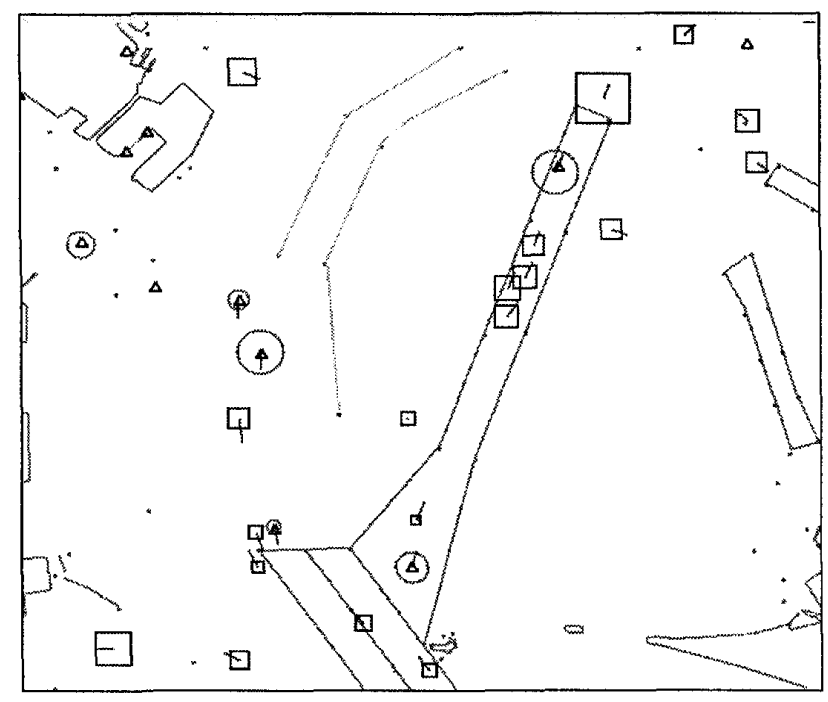

Fig.15 AIS-Radar target checking Screen

- $\square$ : radar only targets.

- $\bigcirc$ : AIS-Radar targets.

- $\triangle$ : AIS targets.
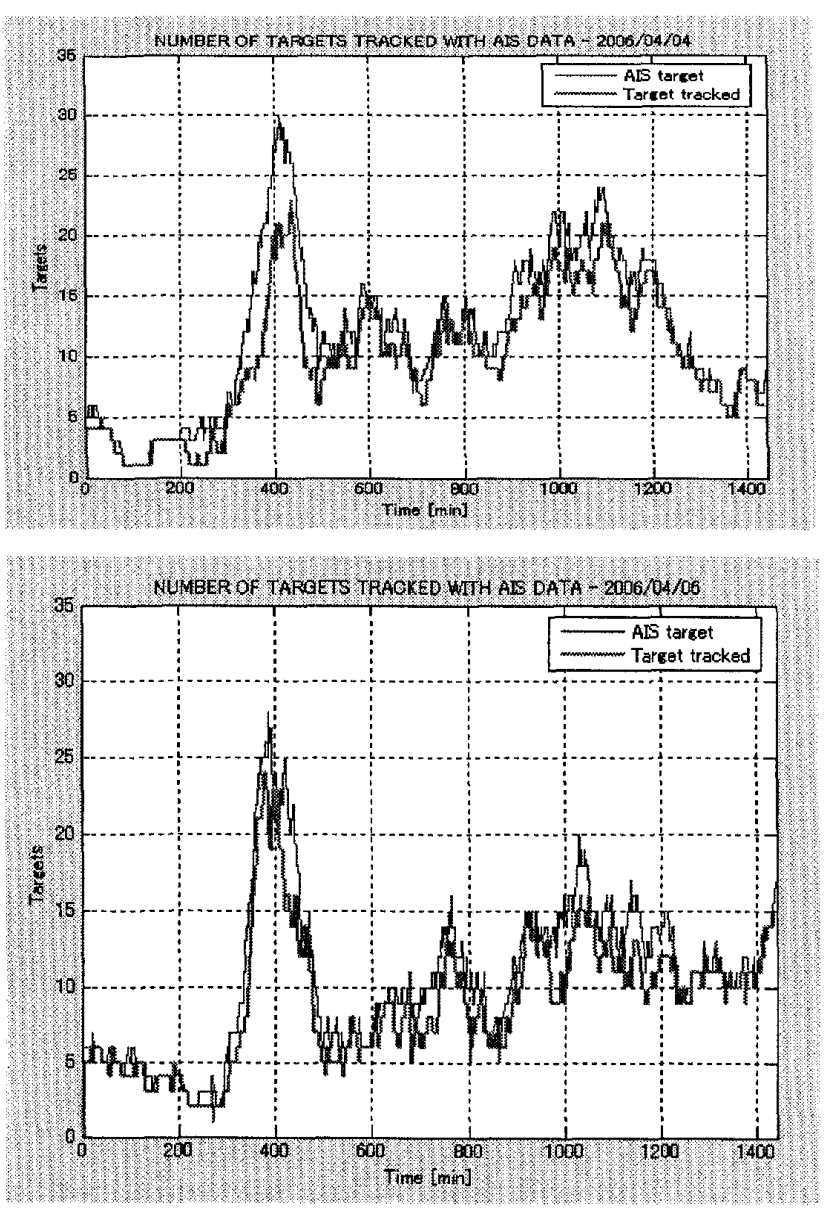

Fig.16 AIS-Radar targets tracking results

\section{Conclusions}

In this research, the Relating-Function has been introduced and used in a structured manner, giving rather promising results. Applying the approach, the efficiency of the automatic tracking has been improved to about $70 \%$, without AIS data provided.

For vessels equipped with AIS, AIS data can be used to achieve better tracking accuracy and reliability. The radar data, conversely, can be used to check the accuracy of received AIS data. With supplemental AIS data, more than $80 \%$ of AIS moving target can be tracked stably. The approach with AIS data will be very useful as the requirements of carrying class B AIS are coming into effects.

However, in order to reach a virtual automatic tracking, some remaining problems still need solving in auto-tracking program.

Firstly, it is the stability of auto-tracking in extreme conditions or when several targets overlap.

Secondly, there need to be a better function of relationship between a lost target and a newly defined one, so as to ensure the continuous tracking of the targets.

\section{References}

(1)HAGIWARA Hideki, SHOJI Ruri, TAMARU Hitoi, LIU Shun, OKANO Tadashi, Development of Remote Radar/AIS Network System for Observing and Analyzing Vessel Traffic in Tokyo Bay.

(2) LIU Shun, HAGIWARA Hideki, SHOJI Ruri, TAMARA Hitoi, New method for Analyzing Traffic Flow Using Integrated Radar Network System and AIS in Tokyo Bay.

\section{Question and answer}

FUKUTO JUNJI (National Maritime Research Institute):

Could you explain the conditions for judging whether automatic tracking is performed or not?

NGUYEN Minh Duc:

Tracking performance can be visually checked using Tracking Screen in Figure 8. Then, to ensure the accuracy, tracking results are compared to manual tracking results, as shown in Figure 9.

Condition for confirming the existence of a moving target is Relating-Value of the 3 image positions to be less than -0.85 .

Condition for taking a position as position of a target is that the Relating-Value of 1 and 2 steps ahead positions of the target and the position concerned to be less than -0.6 . 